https://doi.org/10.11646/zootaxa.4323.2.12

http://zoobank.org/urn:lsid:zoobank.org:pub:07F176A4-FC26-4A3E-8DDE-B03E29DAB799

\title{
Observations on two Procamallanus (Spirocamallanus) species (Nematoda: Camallanidae) from freshwater fishes in Argentina, including description of Procamallanus (Spirocamallanus) juana sp.nov.
}

\author{
GERALDINE RAMALLO ${ }^{1} \&$ LORENA GISELA AILÁN-CHOKE 2,3 \\ ${ }^{1}$ Instituto de Invertebrados, Fundación Miguel Lillo, Miguel Lillo 251, (4000) San Miguel de Tucumán, Argentina. \\ E-mail: gramallosl@yahoo.com.ar \\ ${ }^{2}$ Instituto para el Estudio de la Biodiversidad de Invertebrados, Facultad de Ciencias Naturales, Universidad Nacional de Salta, Av. \\ Bolivia 5150, (4400) Salta, Argentina. E-mail: lorenaailanchoke@gmail.com \\ ${ }^{3}$ Consejo Nacional de Investigaciones Cientificas y Técnicas (CONICET), Argentina
}

\begin{abstract}
Helminthological examination of specimens of the freshwater fishes Pimelodus albicans Valenciennes (Pimelodidae), Pimelodella gracilis (Valenciennes) (Heptapteridae) and Hyphessobrycon anisitsi Eigenmann (Characidae), collected from the Salado River, province of Santiago del Estero, Argentina, revealed the presence of two species of parasitic nematodes, Procamallanus (Spirocamallanus) juana sp. nov. and Procamallanus (Spirocamallanus) hilarii (Camallanidae). Procamallanus (Spirocamallanus) juana sp. nov. was characterized by the presence of six visible pores located around the oral opening in both sexes, 14 cephalic papillae, buccal capsule with 5-7 spiral thickenings, three pairs of preanal papillae, and three pairs of postanal papillae, and conspicuous and pre-equatorial vulva in females. Procamallanus (Spirocamallanus) hilarii was recorded for the first time in Hyphessobrycon anisitsi from northern Argentina. Moreover, zoogeographical and host information for the genus Procamallanus (Spirocamallanus) is summarized.
\end{abstract}

Key words: Procamallanus (Spirocamallanus) juana sp. nov., Siluriformes, Salado River, Santiago del Estero

\section{Introduction}

The genus Procamallanus Baylis, 1923 includes parasitic species of marine and freshwater fishes. Based on the morphology of the buccal capsule, Procamallanus represents five subgenera: Procamallanus Baylis 1923, Denticamallanus Moravec\& Thatcher 1997, Spirocamallanus Olsen 1952, Punctocamallanus Moravec \& Scholz 1991, and Spirocamallanoides Moravec \& Sey 1988 (Moravec \& Thatcher 1997). Of these, the first three occur in Neotropical freshwater fishes (Moravec 1998). To date, there are 29 described species of Procamallanus, parasites of freshwater fishes from the Neotropical Realm (Ramallo 2008; Giese et al. 2009).

In Argentina, five species of Procamallanus (Spirocamallanus) have so far been recorded from freshwater fishes: P. (S.) rarus Travassos, Artigas \& Pereira; P. (S.)inopinatus Travassos, Artigas \& Pereira; P. (S.) hilarii Vaz \& Pereira; P.(S.) huacraensis Ramallo and P.(S.) pintoi, Kohn \& Fernandes (Chemes \& Takemoto 2011; Ramallo 2008; Ailan et al. 2014). The first two species were recorded in siluriform (Pimelodidae and Heptateridae) and characiform (Bryconidae, Characidae, Anostomidae and Serrasalmidae) fishes from the Middle Parana River (Corrientes province). Procamallanus $(S$.) huacraensis, $P$. (S.) pintoi and $P$. (S.) hilarii were registered only in northwestern Argentina. Both P.(S.) huacraensis and $P$. (S.) pintoi were recorded in specimens belonging to Trichomycteridae family (Siluriformes), the first one ( $P$. (S.) huacraensis) was found in Huacra and Vis-Vis Rivers, and Agua Fresca Dike (Catamarca province) and $P$. $(S$.$) pintoi in an irrigation ditch tributary of the Yacones River$ (Salta province). However, Procamallanus (S.) hilarii presented a broader host spectrum (Characiformes, Siluriformes and Cyprinodontiformes) with a more generalized geographical distribution, stretching from the Itaú River, located on the border between to Salta and Bolivia, to the Rio Hondo Dam (Santiago del Estero province) (Antelo et al. 2016). 
During parasitological surveys performed on fishes from the River Salado, province of Santiago del Estero, we isolated specimens of Procamallanus (Spirocamallanus) hilarii for the first time in H. anisitsi (Characidae) and specimens of a new species of the genus Procamallanus Baylis, 1923; subgenus Spirocamallanus Olsen, 1952; which is described herein, from $P$. albicans and P. gracilis. The new species is described, and we expand the host and geographical spectrum of $P$. (S.) hilarii. The geographical implications and host information of the species belonging to the subgenus, Spirocamallanus, are also discussed.

\section{Materials and methods}

During November 2012, March and May 2013; four specimens of Pimelodus albicans, standard length $5.1-7.3 \mathrm{~cm}$ and weight 2.3-8.2 g.; four of Pimelodella gracilis, standard length $2.4-7.7 \mathrm{~cm}$ and weight $2.3-7.8 \mathrm{~g}$ and seven specimens of Hyphessobrycon anisitsi, standard length 3.8-4.7 cm and weight 1.30-2.30 g., were collected from

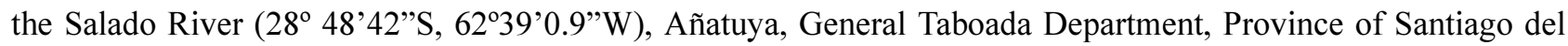
Estero, Argentina. The fishes were fixed in $4 \%$ formaldehyde for $24 \mathrm{hs}$. and stored in $70 \%$ ethanol for the posterior helminthological examination. All nematodes founded were cleared in lactophenol and examined with a light microscope. Drawings were made with the aid of a LEICA microscope. For examination by SEM (scanning electronic microscopy), some specimens from P. albicans and P. gracilis were dehydrated through an ethanol series, acetone, and ether. The specimens were coated with gold and examined with a Zeiss Supra 55VP SEM. Prevalence and mean intensity of infection were calculated based on the definitions of Bush et al. (1997). All measurements are given in millimeters unless otherwise stated.

Type specimens and specimens of $P$. (S.) hilarii have been deposited in the Colección Helmintológica Fundación Miguel Lillo (CH-N-FML), Miguel Lillo 251, (4000) San Miguel de Tucumán, Argentina. The fishes examined were deposited in the Colección Ictiológica Fundación Miguel Lillo (CI-FML), Miguel Lillo 251, (4000) San Miguel de Tucumán.

\section{Order Spirurida Chitwood, 1933}

Family Camallanidae Railliet \& Henry, 1915

Genus Procamallanus Baylis, 1923

Subgenus Spirocamallanus Olsen, 1952

Procamallanus (Spirocamallanus) juana sp. nov.

(Figs 1-2)

Type material. Holotype: female CH-N-FML \#07729; allotype: male CH-N-FML \#07730; paratypes (four females, five males) CH-N-FML \#07731.

Type host. Pimelodus albicans Valenciennes (Siluriformes, Pimelodidae), Ichthyology Collection CI-FML \#6467, collected 30 May 2013.

Additional host: Pimelodella gracilis (Valenciennes) (Siluriformes, Heptateridae), Ichthyology Collection CIFML \#6519, collected 30 May 2013.

Type locality. Salado River, Añatuya, General Taboada Department, Province of Santiago del Estero (28 48'42”'S, 62³9'0.9”W), Northwest Argentina.

Etymology. The new species is named in honor of the memory of Juana Rosa Bennasar de Herrera ("Ms. Monona"), colleague, friend and for years Director of Zoology Area, FML.

Site of infection.Intestine.

Infection parameters. For P. albicans, prevalence: 75\% (3/4); media intensity: 3.3 nematodes per fish and for P. gracilis, $75 \%$ (3/4); 4 nematodes per fish, respectively.

Measurements. Table 1. 
TABLE 1. Measurements of Procamallanus (Spirocamallanus) juana sp. nov. Given in mm, mean \pm SD (minimum value-maximum value). (* Measurement of total length of tail, including the spike).

\begin{tabular}{|c|c|c|c|c|}
\hline \multirow[t]{2}{*}{ Character } & \multirow{2}{*}{$\begin{array}{l}\text { Holotype } \\
+1\end{array}$} & \multirow{2}{*}{$\frac{\text { Allotype }}{\delta 1}$} & \multicolumn{2}{|l|}{ Paratypes } \\
\hline & & & 94 & 35 \\
\hline Total body length & 22,23 & 10.28 & $21.19 \pm 4.31(15.46-24.25)$ & $10.69 \pm 1.51(8.35-12.00)$ \\
\hline Body width & 0.35 & 0.16 & $0.31 \pm 0.05(0.27-0.37)$ & $0.17 \pm 0.02(0.15-0.20)$ \\
\hline Buccal capsule length & 0.06 & 0.053 & $0.06 \pm 0.00(0.06)$ & $0.05 \pm 0.00(0.05)$ \\
\hline Buccal capsule width & 0.06 & 0.05 & $0.06 \pm 0.00(0.06)$ & $0.05 \pm 0.00(0.05-0.06)$ \\
\hline Cephalic ring length & 0.013 & 0.01 & $0.01 \pm 0.00(0.01)$ & $0.01 \pm 0.00(0.01)$ \\
\hline Cephalic ring width & 0.033 & 0.033 & $0.03 \pm 0.00(0.03-0.04)$ & $0.03 \pm 0.00(0.02-0.03)$ \\
\hline Muscular esophagus total length & 0.63 & 0.35 & $0.60 \pm 0.03(0.56-0.63)$ & $0.54 \pm 0.02(0.51-0.56)$ \\
\hline Muscular esophagus total width & 0.07 & 0.06 & $0.07 \pm 0.00(0.07)$ & $0.06 \pm 0.00(0.05-0.06)$ \\
\hline Glandular esophagus total length & 0.94 & 0.87 & $0.84 \pm 0.11(0.70-0.93)$ & $0.82 \pm 0.11(0.70-0.93)$ \\
\hline Glandular esophagus total width & 0.06 & 0.05 & $0.06 \pm 0.00(0.06)$ & $0.05 \pm 0.00(0.04-0.05)$ \\
\hline Nerve ring_-anterior end & 0.23 & 0.24 & $0.26 \pm 0.01(0.25-0.26)$ & $0.23 \pm 0.01(0.22-0.23)$ \\
\hline Excretory pore-anterior end & 0.3 & 0.49 & $0.37 \pm 0.04(0.32-0.40)$ & $0.42 \pm 0.07(0.35-0.49)$ \\
\hline Deirids & - & 0.1 & - & $0.12 \pm 0.03(0.10-0.18)$ \\
\hline Vulva - anterior end & 15.00 & & $14.79 \pm 2.86(10.50-16.36)$ & - \\
\hline Right spicule & - & 0.75 & - & $0.79 \pm 0.02(075-0.80)$ \\
\hline Left spicule & - & 0.27 & & $0.27 \pm 0.01(0.26-0.28)$ \\
\hline Caudal alae length & - & 0.32 & - & $0.34 \pm 0.04(0.30-0.40)$ \\
\hline Tail length* & 0.12 & 0,14 & $0.12 \pm 0.01(0.11-0.12)$ & $0.14 \pm 0.03(0.12-0.20)$ \\
\hline Spike length & 0.02 & - & $0.02 \pm 0.00(0.02)$ & - \\
\hline Larvae length & 0.26 & - & $0.28 \pm 0.06(0.20-0.32)$ & - \\
\hline
\end{tabular}

General description. Medium-sized nematodes with finely, transversely striated cuticle. Mouth aperture rounded, provided and surrounded by six visible pores, fourteen submedian cephalic papillae arranged in three circles, two circlets (medium and external) with four papillae each; and the inner circlet with six larger papillae. Pair of medium amphids present (Fig. 1D, 2A). Buccal capsule orange-brown, thick-walled, barrel-shaped, slightly longer than wide, with simple well developed basal cephalic ring; wall of middle part of capsule strengthened by conspicuous thickenings appearing in lateral view as drop-shaped, extending anteriorly to anterior margin of capsule. In both sexes, inner surface of capsule provided with several spiral ridges, which may be complete and incomplete (not extending from one lateral margin of capsule to other) (Figs. 1A, B, C). Muscular esophagus somewhat shorter than glandular esophagus; both parts of esophagus slightly expanded near their posterior ends (Figs. 1A, C). Intestine narrow. Deirids, observed only in males; small, simple, with rounded end situated at about mid-way between posterior end of buccal capsule and nerve ring (Figs. 2B, C). Excretory pore located approximately in the middle of the muscular esophagus(Figs. 1A, C). Tail of females pointed and of males conical (Figs. 1F, G).

Adult female (five gravid specimens): Buccal capsule with 5-7 inner spiral ridges (Figs. 1C). Deirids absent. Vulva pre-equatorial with conspicuous lips (Fig. 1E, 2E). Vagina muscular, directed posteriorly from vulva. Uterus containing larvae (Figs. 1E). Tail pointed with terminal spike (Figs. 1F, 2F).

Adult Male (six specimens): Buccal capsule with 5-7 inner spiral ridges (Fig. 1B). Deirids present (Figs. 2B, C). Spicules of similar shape, but very unequal in length, right spicule larger than left, both with pointed distal ends (Fig 1G). Six pairs of sessile caudal papillae: three pairs preanal and three pairs postanal (Figs. 1G, 2D). Gubernaculum absent. Posterior end of body ventrally bent, provided with narrow caudal alae. (Figs. 1G, 2D). Tail conical (Fig 1G).

Remarks. The new species belongs to the genus Procamallanus Baylis, 1923, which is characterized by the presence of a solid buccal capsule continuous with the inner surface smooth or with spiral ridges,spicules usually unequal, and caudal alae and gubernaculum present or absent in males. Three subgenera occur in Neotropical 
freshwater fishes. The new species herein described belongs to the subgenus Spirocamallanus Olsen, 1952, because both males and females present spiral thickenings (ridges) in the buccal capsule and the base of the male capsule without teeth (Moravec 1998).Between the species of Procamallanus (Spirocamallanus) recorded for the Neotropical Region, it is possibly distinguished as a morphological group that present caudal alae and unequal spicules, represented by P.(S.) rarus, P.(S.) solani Pinto, Fabio, Noronha \& Rolas, P. (S.) paraguayensis (Petter), P.(S.) freitasi (Moreira, Oliveira \& Costas), P.(S.) pimelodus Pinto, Fábio, Noronha \& Rolas, P.(S.) dessetae (Petter, Golvan \& Tcheprakoff), P.(S.) rebecae (Andrade-Salas, Pineda-Lopez \& Garcias-Magaña) and the new species herein described.

The general morphology of the new species is very similar to $P$. $(S$.) pimelodus; both species are characterized by possessing three pairs of preanal papillae and caudal alae in males, the right spicule exceeds $0.70 \mathrm{~mm}$ and the vulva is pre-equatorial. However, both species differ in the number of cephalic papillae (14 vs. 8); the presence or absence of pores around the mouth aperture; the number of pairs of postanal papillae (3 vs. 8) and the shape of the female tail (pointed with a spike vs. conical without spike). By the presence of caudal alae in males, the new species also resembles $P$. (S.) rarus. Moreover both species were found in freshwater catfishes from Argentina. However, these species differ in that, $P$. (S.) juana sp. nov. has sessile caudal papillae, while those of $P$.(S.) rarus are pedunculated; the vulva in the new species is pre-equatorial while in $P$. $(S$.) rarus it is equatorial, and the spicules are simple in $P .(S$.) juana sp. nov. While $P .(S$.) rarus has unequal and dissimilar spicules, the large spicule with a small ventral outgrowth and the small spicule simple.

The presence of a digit-like projection provided with one or more small terminal cuticular spikes on the female tail, is a feature that the new species shares with some species of Procamallanus (Spirocamallanus), parasites of freshwater and marine fishes (Moravec et al. 2000). In the Neotropical Realm some species have this character. These include P. (S.) rebecae; P. (S.) solani; P. (S.) penneri (Fusco \& Brooks); P. (S.) gobiomori Moravec, SalgadoMaldonado \& Caspeta-Mandujano, 2000; and P. (S.) jaliscensis Moravec, Salgado-Maldonado \& CaspetaMandujano, 2000; all of them are parasites of freshwater fishes (Moravec 1998; Moravec et al. 2000). These species can be distinguished from $P$. (S.) juana sp.nov. by a series of morphological features (such as the number of spiral thickenings in the buccal capsule and the length of right spicule) and the geographical distribution.

Most of the species of Procamallanus (Spirocamallanus) have eight cephalic papillae arranged in two circlets. Nevertheless, the new species is the first in presenting fourteen cephalic papillae organized in three circlets, the external and median circlet formed by four papillae and the inner by six papillae, the papillae of the inner circlet distinctly large. Procamallanus (Spirocamallanus) halitrophus (Fusco \& Overstreet), P. (S.) rebecae, P.(S.) rigbyi Yooyen, Moravec \& Wongsawad and P.(S.) similis Yooyen, Moravec \& Wongsawad also have numerous cephalic papillae, all present 12 papillae organized in three circlets formed by four papillae each and the papillae of the external circlet are distinctly large (Cardenas \& Lanfredi, 2005; Yooyen et al. 2011). Nevertheless these species differ mainly in the host range and the geographical distribution. The first two species were recorded in the Neotropical Realm. Procamallanus (Spirocamallanus) halitrophus was record in marine fishes from the northern Gulf of Mexico and the coasts of Rio de Janeiro, Brasil; while $P$. (S.) rebecae was found in cichlid freshwater fishes from southern Mexico (Moravec, 1998). In contrast to $P$. (S.) rigbyi and $P$. (S.) similis that were recorded in marine perciform fishes from the Gulf of Thailand (Yooyen et al. 2011).

By the presence of pores surrounding the margin of the oral opening, $P$. (S.) juana sp. nov. resemble $P .(S$.) daleneae (Boomker), $P$. (S.) spiralis Baylis and $P$. (S.) serranochromis Moravec \& Van As. The first two present six pores, which coincides with the new species; while $P$. (S.) serranochromis has four pores in the cephalic end. Nevertheless, these species can be distinguished mainly from $P$. (S.) juana sp. nov. by the geographical distribution. Procamallanus $(S$.) daleneae and $P$. (S.) serranochromis were only recorded in African freshwater fishes. While, Procamallanus (S.) spiralis was also found in Africa and in different species of marine fishes in the Gulf of Suez, off the Atlantic and Pacific coasts of Mexico, in Philippine and Indonesian waters (off the Celebes), and in the Indian Ocean off the coast of Pakistan and India (Moravec \& Van As 2015).

Procamallanus (S.) juana sp. nov. can be distinguished from all congeners, by possessing 14 cephalic papillae arranged in three circlets (one of six papillae and two remaining with four papillae); six pores distinctly surrounding the mouth aperture; a buccal capsule with 5-7 spiral ridges; three pairs of preanal and three pairs of postanal papillae in males and a terminal cuticular spike on the female tail. Thus in this paper, we propose the erection of the new species of Procamallanus (Spirocamallanus), which is the $30^{\text {th }}$ species of this genus for Neotropical Realm and the sixth for Argentina. 

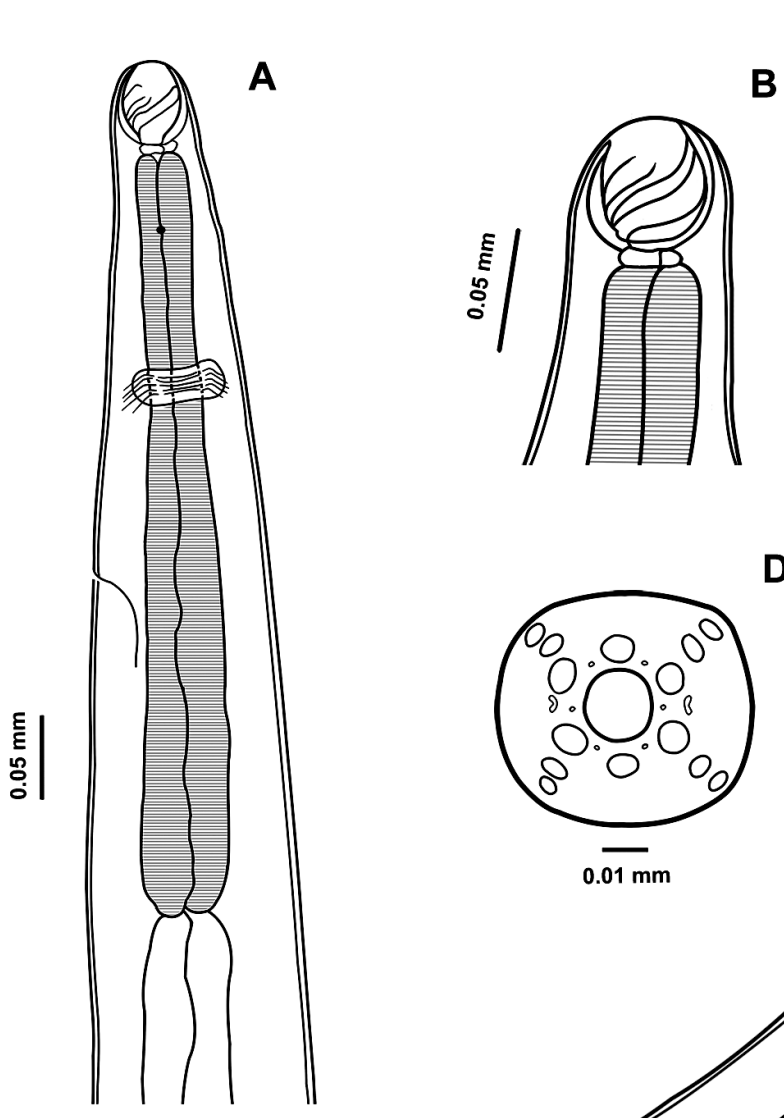

B

C
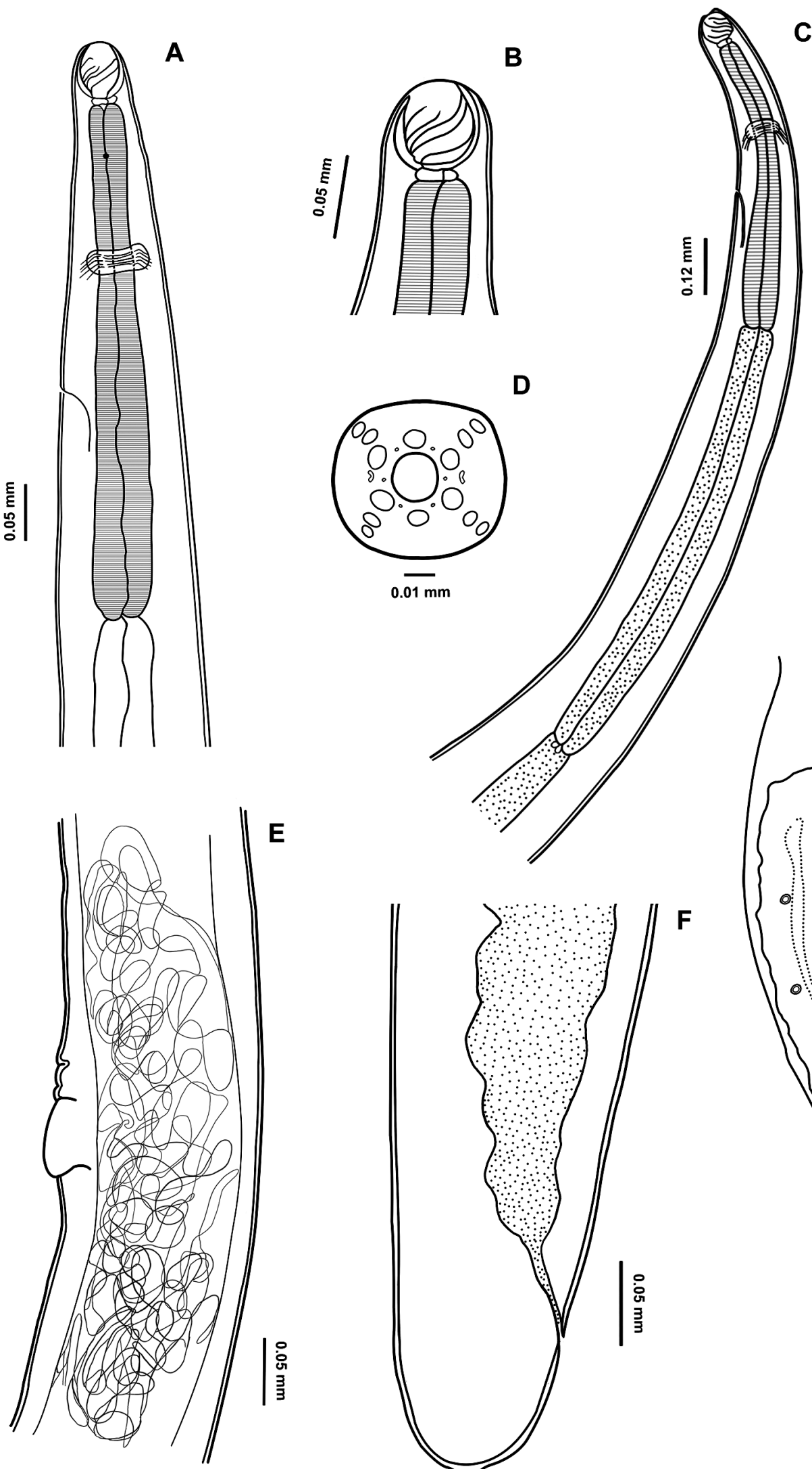

E
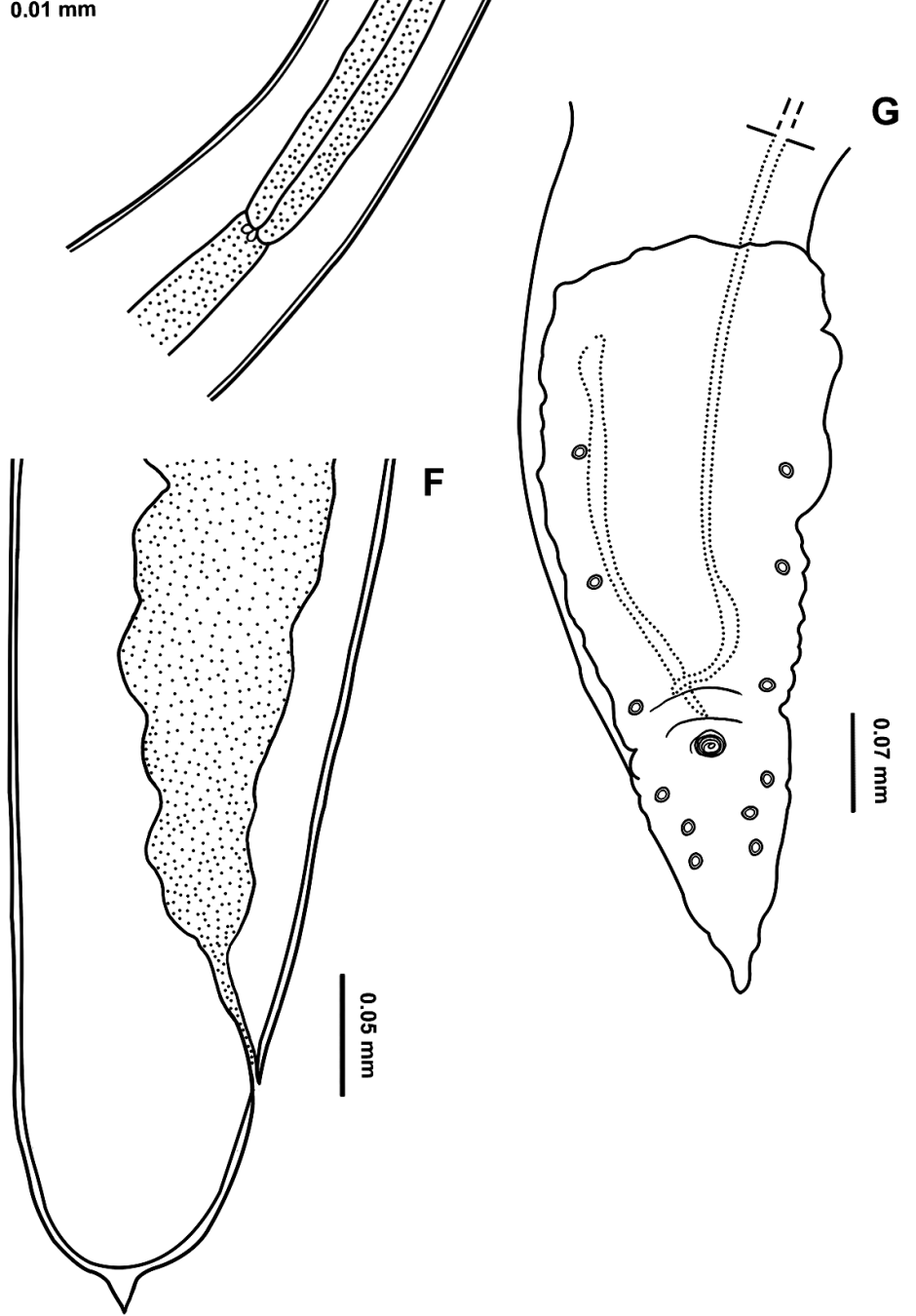

FIGURE 1. Procamallanus (Spirocamallanus) juana sp. nov. (A) Male, anterior end, lateral view. (B) Male, head, lateral view. (C) Female, anterior end, lateral view. (D) Female, apical view. (E) Female, vulva, lateral view. (F) Female, tail, lateral view. (G) Male, posterior end with spicules and papillae, ventral view. 


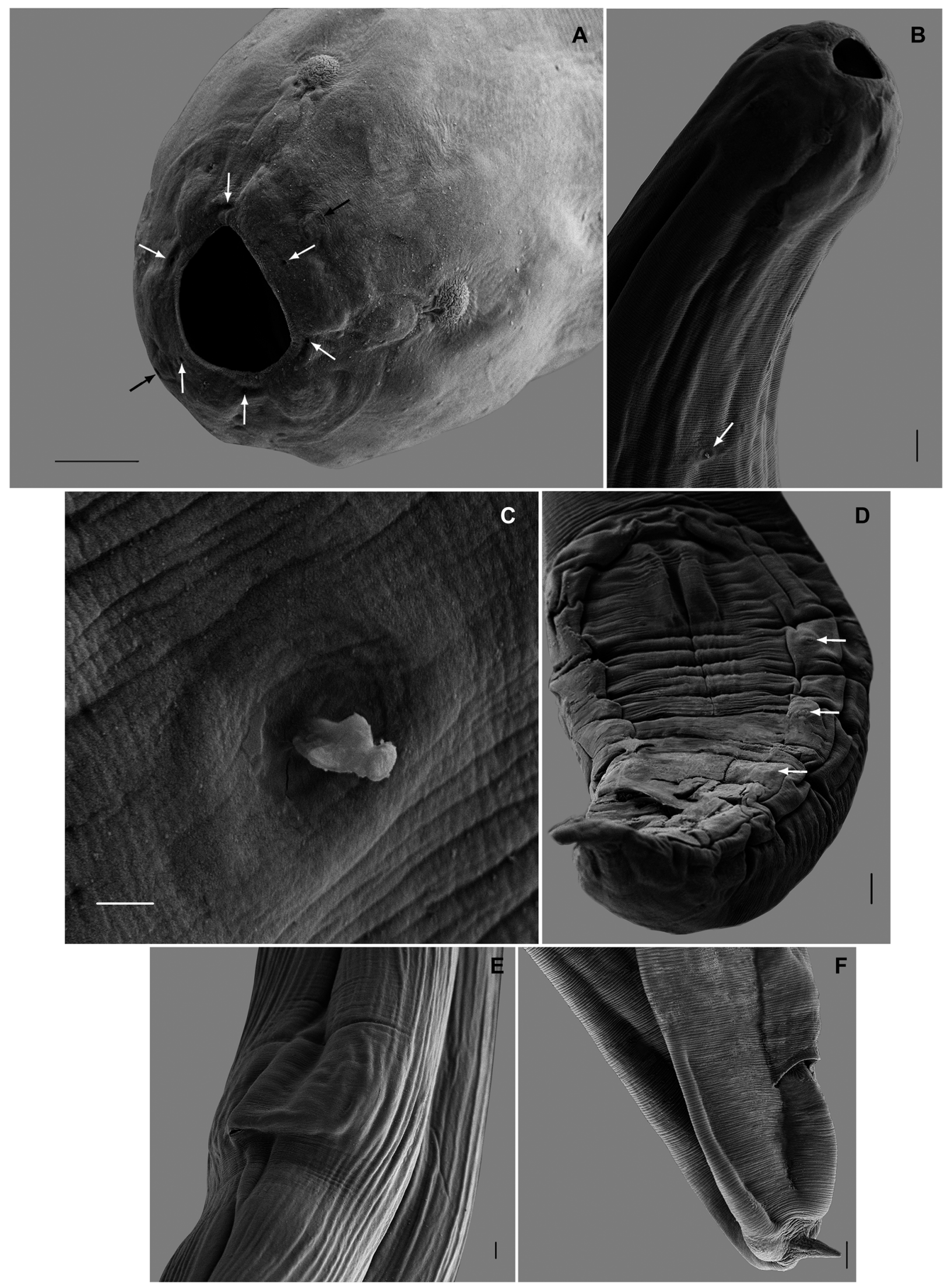

FIGURE 2. Procamallanus (Spirocamallanus) juana sp. nov. (SEM micrographs) (A) Male, cephalic end. Six pores (white arrows), amphids (black arrows). Scale $=10 \mu \mathrm{m}$. (B) Anterior end of male, sublateral view. Deirid (white arrow). Scale $=10 \mu \mathrm{m}$. (C) Detail deirid. Scale $=1 \mu \mathrm{m}$. (D) Posterior end of male, ventral view. Preanal papillae (white arrows). Scale $=10 \mu \mathrm{m}$. (E) Female, vulva, subventral view. Scale $=10 \mu \mathrm{m}$. (F). Female, posterior end, lateral view. Scale $=10 \mu \mathrm{m}$. 
Procamallanus (Spirocamallanus) hilarii Vaz \& Pereira, 1934

Specimens examined. Six males and one female CH-N-FML \#7732 isolated from Hyphessobrycon anisitsi (Eigenmann, 1907) (CI-FML \#6429) from Salado River, Añatuya, General Taboada Department, Province of Santiago del Estero (28 48'42”S, 62³9'0.9”W), Northwest Argentina.

Infection parameters. Prevalence: $57 \%(4 / 7)$, mean intensity 1.75 nematodes per fish.

Diagnosis. Medium nematodes with transversely striated cuticle. Buccal capsule with 13-18 spiral thickenings, basal ring well development. Muscular esophagus claviform, much shorter than glandular esophagus. Male with three pairs of preanal, 1-2 pairs adanal and three pairs of postanal papillae. Gubernaculum absent. Spicules short and subequal. Larger spicule 0.070-0.082, small spicule 0.050-0.062. Tail conical.In females, vulva in middle of body; uterus containing larvae. Tail rounded with terminal caudal appendix.

Remarks. The morphological and morphometric analyses of the specimens analyzed by light microscopy, allowed the identification of Procamallanus (Spirocamallanus) hilarii, agreeing with the original description. Procamallanus $(S$.) hilarii belongs to a morphological group of species without caudal alae and with short and similar spicules in males, represented by $P$. (S.) chimusensis (Freitas \& Ibañez), P. (S.) inopinatus Travassos, Artigas \& Pereira, P. (S.) krameri (Petter), P. (S.) neocaballeroi (Caballero-Deloya), P. (S.) paraensis Pinto \& Noronha, $P$. (S.) pintoi (Khon \& Fernandes) and P. (S.) saofranciscencis (Moreira, Oliveira \& Costas) (Moravec et al. 2004).

By the general morphology, Procamallanus $(S$.) hilarii is most closely related with P. (S.) neocaballeroi. Both species present numerous spiral ridges (13-19) occupying the whole inner surface of the buccal capsule and a glandular esophagus at least three times longer than muscular esophagus. Procamallanus $(S$.) neocaballeroi can be readily distinguished from Procamallanus (S.) hilarii by possessing equal spicules and four pairs of preanal papillae and six pairs of postanal papillae. Moreover these species differ in the geographical distribution in the Neotropical Region. Procamallanus (S.) hilarii was recorded for Brazil, Perú and Argentina; while P. (S.) neocaballeroi has only been recorded in Mexico (Moravec 1998).

Discussion. The genus Procamallanus Baylis, 1923 comprises numerous species described in a broad variety of host and geographical regions, in both marine and freshwater fishes. Although, many authors (Andrade-Salas et al. 1994) consider Spirocamallanus Olsen, 1952 as a distinct genus, Moravec \& Sey (1988) consider Spirocamallanus a subgenus of Procamallanus to accommodate the species where both males and females have the buccal capsule with spiral ridges. Nevertheless, it is clear that this taxonomic system based on the morphology of the buccal capsule is more or less artificial and does not reflect the phylogenetic relationships (Moravec \& Thatcher 1997).

Andrade-Salas et al. (1994) proposed an alphabetic list with all nominal species grouped according to the geographical zones. However, this list is outdated, because later new species of Procamallanus were described. With respect to the Neotropical Realm, species of Procamallanus (Spirocamallanus) occur both in marine and continental water systems. To date, there are 29 species recorded from freshwater fishes, and they are widely distributed for the Neotropical Realm; mainly in Mexico, Lesser Antilles, Venezuela, Brasil, Paraguay, Perú and Argentina.

In Argentina, there are five species of Procamallanus (Spirocamallanus) ( $P$. (S.) inopinatus, $P$. (S.) rarus, P. (S.) huacraensis, P.(S.) hilarii and P. (S.) pintoi) recorded from characiform and siluriform freshwater fishes, mainly distributed in the regions northwest and northeast of the country. In the province of Corrientes, northeast region, it was registered $P$. (S.) inopinatus in Totora y Perez Lagoon, Riachuelo basin, and Riachuelo River Lagoon; and P. (S.) rarus in Parana River. Procamallanus (S.) inopinatus presents a wide host range. It was recorded from specimens of Characiformes (Brycon orbignyanus Valenciennes, Poptella paraguayensis Eigemann, Leporinus maculatus Müller \& Troschel, Serrasalmus marginatus Valenciennnes, S. spilopleura Kner, Pygocentrus nattereri Kner) and Siluriformes (Luciopimelodus pati Valenciennes and Pseudoplatysoma corruscans Spix \& Agassiz). By contrast, P. (S.) rarus was only found in P. albicans (Chemes \& Takemoto 2011). Moreover, there are many records of both species in Brasil. Procamallanus $(S$.) inopinatus is also recorded from Paraguay and Venezuela and $P$. (S.) rarus from Perú (Moravec 1998). The new species herein described is most closely related with $P$. (S.) rarus by the presence of caudal alae and both species were found in the same host, $P$. albicans.

With respect to the northwest region, there are three species recorded: $P$. (S.) huacraensis, $P$. $(S$.) pintoi and $P$. (S.) hilarii. The first two were found only in catfishes: Trichomycterus corduvensis and T. spegazzini respectively 
(All Trichomycteridae). Procamallanus. (S.) huacraensis was registered from Huacra and Vis-Vis Rivers, and Dike Agua Fresca (Catamarca Province). While, there is a unique record of $P$. (S.) pintoi in irrigation ditch tributary of River Yacones (Vaqueros municipality, Salta Province). Procamallanus $(S$.) hilarii was recorded in a wide host spectrum: Salminus brasiliensis Cuvier (=S. maxillosus Valenciennes), Megaleporinus obstusidens Valenciennes (=Leporinus obstusidens Valenciennes), Oligosarcus jenynsii Günther, Hoplias malabaricus Bloch, A. abramis Jenyns, A. lacustris Lutken, A. rutilus Jenyns, Piabina thomasi Fowler (all characids); Pimelodus albicans Valenciennes (Pimelodidae, Siluriformes) and Jenynsia alternimaculata Fowler (Anablepidae, Cyprinodontiformes). Its geographical distribution includes dam, rivers, streams and tributaries from several localities of the provinces of Tucumán, Santiago del Estero and Salta (Northwest Argentina) (Ailan et al. 2014; Antelo et al. 2016; Ramallo 2008). Procamallanus (S.) juana sp. nov. was also found in P. albicans. Thus both species $(P$. $(S$.) juana sp. nov. and $P$. (S.) hilarii) share the same host species and present records in the province of Santiago del Estero. However, these species differ in their general morphology, mainly in the presence of caudal alae, the number of spiral ridges in the buccal capsule and the number of caudal papillae in males (Moravec 1998).

With the new finding, the number of species of Procamallanus (Spirocamallanus) was extended for the Neotropical Realm. Moreover, $P$. (S.) hilarii extends its host range and geographical distribution.

\section{Acknowledgments}

Thanks to Fundación Miguel Lillo for providing financial support and the Secretaria de Medio Ambiente del Gobierno de la Provincia de Santiago del Estero for allowing us to make the collection of ichthyologic materials. We would also like to thank Fabiana Cancino, for their assistance in the capture of hosts, and her help its identification. Pablo Pereyra helped us in the digitalization of figures and Luciano Martinez with the staff of the Laboratory of Scanning Electron Microscopy (CIME), for their technical assistance.

\section{References}

Ailán Choke, L., Ramallo, G., Nieva, L. \& Davies, D. (2014) Helmintos parásitos de peces fluviales, provincia de Salta, Argentina. Acta Zoológica Lilloana, 58, 251-255. https://doi.org/10.15560/10.3.597

Antelo, C., Bulacio, E., Cancino, F., Marigliano, N., Peralta, M., Ramallo, G. \& Romero, F. (2016) Biodiversidad y Fronteras: Cuenca del Río Bermejo (Salta, Argentina).Serie Conservación de la Naturaleza $N^{\circ} 21$. Fundación Miguel Lillo, San Miguel de Tucumán, 82 pp. https://doi.org/10.14522/darwiniana.2014.21.560

Andrade-Salas, O., Pineda-Lopez, R.F., Garcia-Magaña, L. (1994) Spirocamallanus rebecae sp. n. (Nematoda: Camallanidae) from freshwater fishes in south-eastern Mexico. Folia Parasitologica, 41, 259-270. https://doi.org/10.1515/ap-2015-0032

Bush, A.O., Lafferty, K.D., Lotz, J.M. \& Shostak, A.W. (1997) Parasitology meets ecology on its own terms: Margolis et al. revisited. Journal of Parasitology, 83, 575-583. https://doi.org/10.2307/3284227

Cardenas, M.Q. \& Lanfredi, R.M. (2005) Futher description of Procamallanus (Spirocamallanus) halitrophus comb. n. (Nematoda: Camallanidae) from flounder off the Brazilian coast by light and scanning microscopy electron microscopy. Journal of Parasitology, 91, 606-613. https://doi.org/10.1645/ge-3422

Chemes, S.B. \& Takemoto, R.M. (2011) Diversity of parasites from Middle Paraná systemfreshwater fishes, Argentina.International Journal of Biodiversity and Conservation, 3, 249-266. https://doi.org/10.1023/b:bioc.0000035870.36495.fc

Giese, E.G., Santos, J.N. \& Lanfredi, R.M. (2009) A New Species of Camallanidae from Ageneiosus ucayalensis (Pisces: Siluriformes) from Pará State, Brazil. Journal of Parasitology, 95 (2), 407-412. https://doi.org/10.1645/ge-1680.1

Moravec, F. (1998) Nematodes of freshwater fishes of the Neotropical Region. Academy of Sciences of the Czech Republic, Praha, $464 \mathrm{pp}$.

Moravec, F. \& Sey, O. (1988) Nematodes of freshwater fishes from North Vietnam.Part 1.Camallanoidea and Habronematoidea. Acta Societatis Zoologicae Bohemoslovacae, 52, 128-148. https://doi.org/10.14411/fp.2017.010

Moravec, F. \& Thatcher, V.E. (1997) Procamallanus (Denticamallanus subgen. n.) dentatus n. sp. (Nematoda: Camallanidae) 
from the characid fish, Bryconops alburnoides, in the Brazilian Amazon. Parasite, 4, 239-243.

https://doi.org/10.1051/parasite/1997043239

Moravec, F., Salgado-Maldonado, G. \& Caspeta-Mandujano, J. (2000) Three new Procamallanus (Spirocamallanus) species from freshwater fishes in Mexico.Journal of Parasitology, 86, 119-127.

https://doi.org/10.2307/3284921

Moravec, F., Chara, J. \& Shinn, A.P. (2004) Two nematodes, Dentinema trichomycteri n. g., n. sp. (Cosmocercidae) and Procamallanus chimusensis Freitas \& Ibáñez, 1968 (Camallanidae), from catfishes Trichomycterus spp. (Pisces) in Colombia. Systematic Parasitology, 59, 189-197.

https://doi.org/10.1023/b:sypa.0000048098.80098.26

Moravec, F. \& Van As, L.L. (2015) Procamallanus (Spirocamallanus) spp. (Nematoda: Camallanidae) from fishes of the Okavango River, Botswana, including P. (S.) serranochromis n. sp. parasitic in Serranochromis spp.(Cichlidae). SystematicParasitology, 90,151-164. https://doi.org/10.1007/s11230-014-9542-z

Ramallo, G. (2008) Nueva especie de Procamallanus (Spirocamallanus) (Nematoda, Camallanidae), parásito de Trichomycterus corduvensis (Siluriformes: Trichomycteridae), en el Norte de Argentina. Acta Zoológica Lilloana, 52, 2529. https://doi.org/10.1515/ap-2015-0032

Yooyen, T., Moravec, F. \& Wongsawad, C. (2011) Two new sibling species of Procamallanus (Spirocamallanus) (Nematoda: Camallanidae) from marine fishes in the gulf of Thailand. Journal of Parasitology, 97, 931-938.

https://doi.org/10.1645/ge-2806.1 